\title{
Microstructural Features of Cold-Rolled Carbon Steel Evaluated by X-ray Diffraction Line Profile Analysis and Their Correlation with Mechanical Properties
}

\author{
Masayoshi KUMAGAl, ${ }^{*}$ Muneyuki IMAFUKU and Shin-ichi OHYA \\ Tokyo City University, Mechanical Systems Engineering, 1-28-1, Tamazutsumi, Setagaya-ku, Tokyo, $158-8557$ Japan. \\ (Received on June 11, 2013; accepted on August 21, 2013)
}

\begin{abstract}
Line profile analyses of cold-rolled carbon steels were conducted to evaluate microstructural features such as dislocation density, crystallite size, and $M$ values. After the samples were subjected to $40 \%$ coldrolling, dislocation density increased from $7 \times 10^{13} \mathrm{~m}^{-2}$ to $2 \times 10^{15} \mathrm{~m}^{-2}$ and crystallite size decreased from $155 \mathrm{~nm}$ to $35 \mathrm{~nm}$. The component ratio of screw and edge dislocations was approximately 1:1, as determined from the evaluation of the $q$ values. The $M$ value that indicated interaction of dislocations substantially decreased during the initial stage of cold-rolling, which means interaction of dislocations becomes strong. Proof stress, hardness, and tensile strength were increased by the cold-rolling process. Furthermore, the ratios between proof stress and hardness were initially 2 and increased to approximately 3 . The correlation between the microstructures and the mechanical properties was demonstrated according to the Bailey-Hirsch relationship between flow shear stress and dislocation density. Variations in the proof stress and hardness as a function of the square root of dislocation density indicated that the work-hardening of the material is affected by not only the total amount of dislocations but also other factors, such as crystallite size and arrangement of dislocations.
\end{abstract}

KEY WORDS: dislocation; X-ray diffraction; cold-rolling; work-hardening; mechanical property.

\section{Introduction}

Most metals that are used in the manufacture of mechanical and structural materials are polycrystalline. Their mechanical properties depend on several scales of features such as crystal texture, grain size, and dislocation density. Optical microscopy and scanning electron microscopy are typically used to study relatively large scale of features, whereas transmission electron microscopy (TEM) is used to study nano-scale features. TEM is a particularly useful method because it can directly observe microstructures in nano-scale; TEM specimens, however, must be prepared as thin films. Therefore, TEM is a destructive evaluation method. Furthermore, microstructural features can be altered during the sample preparation procedure.

$\mathrm{X}$-ray diffraction line profiles provide information about microstructures. The characteristics of microstructures can therefore be extracted via analysis of the obtained profiles. ${ }^{1)}$ $\mathrm{X}$-ray diffraction methods are more convenient than TEM methods and are non-destructive. Furthermore, because the characteristics can be obtained as average values for an Xray-irradiated area, microstructural features can be analyzed quantitatively. Quantitative X-ray analysis methods, such as the Williamson-Hall ${ }^{2)}$ and Warren-Averbach methods, ${ }^{3)}$ are well established. However, these methods do not take into account the influence of elastic anisotropy and cannot be

* Corresponding author: E-mail: mkumagai@tcu.ac.jp DOI: http://dx.doi.org/10.2355/isijinternational.54.206 applied to materials with large elastic anisotropy. For instance, the $A$ index $\left(=2\left(S_{11}-S_{12}\right) / S_{44}\right)$, which shows the amplitude of elastic anisotropy, is 1.00 for $\mathrm{W}$, whereas those of $\mathrm{Al}, \mathrm{Fe}$, and $\mathrm{Cu}$ are $1.22,2.36$, and 3.21, respectively. ${ }^{4)} \mathrm{An}$ $A$ index of 1 means the material is isotropic, and deviations from 1 reflect the amplitude of anisotropy. Because the $A$ indices of $\mathrm{W}$ and $\mathrm{Al}$ are close to 1 , they can be analyzed via the above-mentioned line profile methods. $\mathrm{Fe}$ and $\mathrm{Cu}$, however, cannot be analyzed by such methods because of their anisotropy. Consequently, although steels are the most common mechanical and structural materials, they have rarely been evaluated using line profile analysis.

Ungár et al. proposed a method that takes into account the influence of elastic anisotropy and demonstrated this method's applications to elastic anisotropic materials. ${ }^{5)}$ The fundamental studies were conducted using copper and pure iron and their powders. ${ }^{5-8)}$ Common materials such as carbon steels, however, have been rarely studied. Thus, we studied the microstructural features in nano-scale of colddrawn carbon steel and their correlation to the steel's mechanical properties using X-ray diffraction line profile analysis.

\section{Procedure for the Evaluation by X-ray Diffraction}

Measured line profiles exhibit two kinds of broadening: physical broadening due to inhomogeneous strains and 
instrumental broadening due to the measurement system. Instrumental broadening must be eliminated from measured line profiles to determine the intrinsic dislocation density and the crystallite size. In this study, instrumental broadening was eliminated using Voigt function fitting. Measured profiles were fitted by a Voigt function, and then the fullwidths at half-maxima (FWHMs) of instrumental profiles were subtracted from those of measured diffraction profiles. Because a Voigt function is a convolution of a Lorentz function and a Gauss function, physical profiles become a convolution of subtracted Lorentz and Gauss functions. The intrinsic FWHM of the Lorentzian component can be obtained by simple subtraction, and the intrinsic FWHM of the Gaussian component, $B_{\mathrm{p}}$, can be obtained by $B_{\mathrm{p}}=\left(B_{\mathrm{m}}^{2}-B_{\mathrm{i}}^{2}\right)^{1 / 2}$ with the measured and instrumental components, $B_{\mathrm{m}}^{2}$ and $B_{\mathrm{i}}^{2}$. The reconvolution of the obtained physical broadenings, i.e., the Lorentzian and Gaussian components, becomes the physical line profile for the material.

The fundamental equation of the modified WilliamsonHall method ${ }^{5)}$ is

$$
\Delta K \cong \frac{0.9}{D}+\left(\frac{\pi M b^{2}}{2}\right)^{1 / 2} \rho^{1 / 2}\left(K \bar{C}^{1 / 2}\right)+O\left(K^{2} \bar{C}\right),
$$

where $K$ is scattering vector, $\Delta K$ is the widths of line profile, $b$ is the Burgers vector, $M$ is a dimensionless dislocation arrangement parameter, ${ }^{9)} \rho$ is the dislocation density, $D$ is crystallite size, $O\left(K^{2} \bar{C}\right)$ is a higher-order parameter of $K^{2} \bar{C}$, and $\bar{C}$ is the mean value of the contrast factor:

$$
\bar{C}=C_{h 00}\left(1-q H^{2}\right) \text {. }
$$

The term $H^{2}$ is expressed using Miller indices $h k l$ as

$$
H^{2}=\frac{h^{2} k^{2}+k^{2} l^{2}+l^{2} h^{2}}{\left(h^{2}+k^{2}+l^{2}\right)^{2}}
$$

The parameters $C_{h 00}$ and $q$ have different values between screw and edge dislocations. Each value of $C_{h 00}$ and $q$ was calculated using ANIZC, a computational program developed by Borbély et al. ${ }^{10)}$ The slip system of bec is $<111>$ $\{110\}$, and the elastic stiffness parameters of single crystal iron- $C_{11}, C_{12}$, and $C_{44}$-are $228.0,132.0$, and $116.5 \mathrm{GPa}$, respectively. ${ }^{11)}$ The respective values of $C_{h 00}$ and $q$ for a screw dislocation are 0.305 and 2.64 and those for an edge dislocation are 0.263 and 1.29. Because $q$ depends on the component rate of screw and edge dislocations, the actual $C_{h 00}$ and $q$ can be determined by the following equation, which is obtained from Eqs. (1) and (2):

$$
\frac{\Delta K^{2}-\alpha^{2}}{K^{2}}=\beta C_{h 00}\left(1-q H^{2}\right) \text {. }
$$

The parameters $C_{h 00}$ and $q$ are optimized using a linear relation between $H^{2}$ and the left component of Eq. (4), where $\alpha$ and $\beta$ are constants. The dislocation density can be calculated from the inhomogeneous strain, $\langle\varepsilon\rangle$, with contrast factor $\bar{C}$ using the following equation:

$$
\left\langle\varepsilon(L)^{2}\right\rangle \cong\left(\frac{\rho \bar{C} b^{2}}{4 \pi}\right) \ln \left(\frac{R_{e}}{L}\right),
$$

where $R_{e}$ is the effective outer cut-off radius of dislocations. The modified Warren-Averbach equation is expressed with Eq. (5) as

$$
\ln A(L)=\ln A^{S}(L)-\left(\frac{\pi L^{2} \rho b^{2}}{2}\right) \ln \left(\frac{R_{e}}{L}\right)\left(K^{2} \bar{C}\right)+O\left(K^{2} \bar{C}\right)^{2} \ldots
$$

The dislocation density and the crystallite size can be calculated by Eq. (6), where, $A(L)$ is the Fourier coefficient, $A^{\mathrm{S}}(L)$ is the intercept of the plots and indicates the crystallite size, and $L$ is the Fourier length. The dislocation density is obtained from the first-order slope of a graph of $\ln A(L)$ at each $L$ as a function of $K^{2} \bar{C}$. The crystallite size is obtained from the intercept of Eq. (6).

\section{Experimental}

S45C carbon steel was used for this study. Stress-relief annealing was performed at $680^{\circ} \mathrm{C}$ for $5 \mathrm{~h}$, after the specimens were milled to a thickness of $5 \mathrm{~mm}$. Five reduction rates of specimens $(0,10,20,30$, and $40 \%)$ were prepared by cold-rolling.

An X-ray diffractometer (D8 ADVANCE, Bruker AXS) with Bragg-Brentano geometry was used for diffraction profile measurements. An X-ray tube with $\mathrm{Cu}$ target was used, and $\mathrm{Ni}$ filter was used to eliminate the $\mathrm{K} \beta$ line. The 110,200 , 220, 211, 310, and 222 indices were measured. Standard $\mathrm{LaB}_{6}$ powder (SRM 660a, NIST) was used to enable deconvolution of the measured profiles. The microstructural features were analyzed using the previously discussed procedures.

Mechanical properties of the samples, such as their proof stress, tensile strength, and hardness, were also measured. Proof stresses and tensile strengths were examined using a tensile strength tester (AG-Xplus, Shimadzu) and harnesses were examined using a Vickers hardness tester (HMV-1, Shimadzu), respectively.

\section{Results and Discussion}

\subsection{Line Profile Analysis}

Figure 1 shows normalized line profiles after the $\mathrm{K} \alpha_{2}$ line, background, and instrumental components were eliminated. The narrowest line profiles were the initial states of the specimens, and the widths increased in the order corresponding to the rolling reduction rate in all of the diffraction indices. Qualitatively, the dislocation density apparently increased as plastic deformation increased during cold-rolling. The profiles were analyzed by the modified WilliamsonHall/Warren-Averbach methods for quantitative evaluation.

The $q$ values were obtained using Eq. (4) as shown in Fig. 2. Because $q$ depends on the composition ratio of screw and edge dislocations, it can be obtained from the $q$ values. The $q$ values of pure screw and edge dislocations are 2.64 and 1.29 , respectively. In this study, the mean value of $q$ was approximately 2 , which means that screw and edge dislocations occurred in approximately equal numbers. In detail, the $q$ values slightly decreased in samples prepared at reduction rates of $0 \%$ to $30 \%$ and increased in samples prepared at reduction rates of $30 \%$ to $40 \%$. These results indicate that approximately the same amounts of screw and edge dislocations were present in samples prepared at reduction rates from $0 \%$ to $30 \%$ and that the proportion of screw dislocations slightly increased in samples prepared at reduction rates of $30 \%$ to $40 \%$.

We subsequently calculated the dislocation density and 

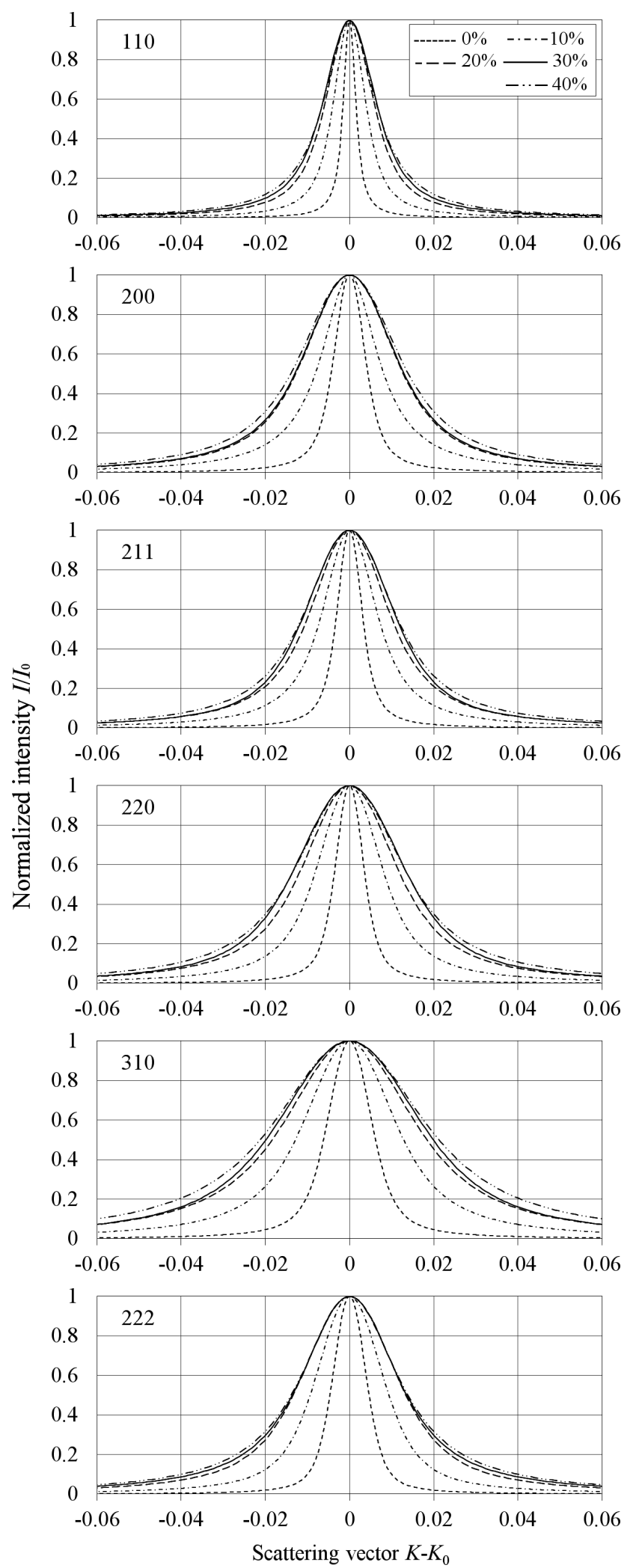

Fig. 1. Measured diffraction profiles. Each graph denotes five steps of rolling reduction rates. The width of the profiles reflects the order of the rolling reduction rates.

crystallite size by Eq. (6) using the obtained contrast factor, $\bar{C}$. In this method, the dislocation density and crystallite size were calculated through Fourier coefficients of the line profiles. Therefore, Fourier series expansions were performed on the obtained line profiles shown in Fig. 1. The obtained Fourier coefficients, $A(L)$, are plotted as functions of the Fourier length, $L$, in Fig. 3. The broadened plots of

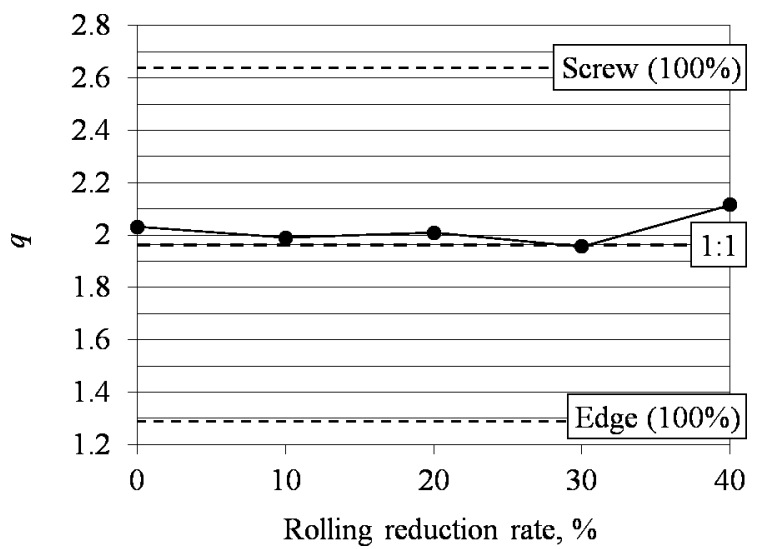

Fig. 2. Component ratios of screw and edge dislocations. The values of 2.64 and 1.29 represent pure screw and edge dislocations, respectively.

Fourier coefficients indicate that coherent domains are large; specifically, the regions with fewer lattice defects are larger because specimens with large coherent domains contain fewer lattice defects. In Fig. 3, the regions corresponding to coherent domains became narrower with an increase in the rolling reduction rate. Therefore, it seems that the dislocation densities increased and crystallite sizes decreased with an increase in the rolling reduction rate.

The dislocation densities and crystallite sizes calculated by line profile analyses are shown in Figs. $\mathbf{4}$ and 5, respectively. The dislocation density before cold-rolling was $7 \times$ $10^{13} \mathrm{~m}^{-2}$ and then increased with an increase in the rolling reduction rate to approximately $2 \times 10^{15} \mathrm{~m}^{-2}$ when the rolling reduction rate was $40 \%$. However, the crystallite size before cold-rolling was $155 \mathrm{~nm}$ and decreased with an increase in the rolling reduction rate to $35 \mathrm{~nm}$ when the rolling reduction rate was $40 \%$. Thus, the dislocation densities increased and the crystallite sizes decreased when the rolling reduction rate was increased. The rate of increase of the dislocation density as a function of the rolling reduction rate was constant. However, the rate of decrease of the crystallite size was not constant. It decreased as the rolling reduction rate was increased and converged at $30-40 \mathrm{~nm}$. Here crystallite size denotes the coherent domain size for X-ray diffraction, which depends on several factors such as sell-structures, subgrain boundaries and low angle boundaries within grains. Therefore, the crystallite size of a material is generally smaller than the crystal grain size.

The dimensionless dislocation arrangement parameter $M$ is calculated as a function of the effective outer cut-off radius of dislocations, $R_{\mathrm{e}}$, and the dislocation density, $\rho$, as $M=$ $R_{\mathrm{e}} \rho^{1 / 2}$; the results of these calculations are shown in Fig. 6. The value of $M$ shows interactive effects between dislocations. When the dislocations arrange randomly, $M$ is relatively large and dislocations have weak interactions. When dislocation dipoles are formed, the value of $M$ typically becomes small. ${ }^{12,13)}$ The $M$ value was 1.6 before cold-rolling and steeply decreased to 0.6 during the first $10 \%$ cold-rolling. The $M$ value then slowly decreased to 0.4 as the rolling reduction rate was increased. We hypothesize that the dislocations aligned and the crystallite domains divided at the same time during the initial step and that the dislocation density subsequently increased without remarkable varia- 



Fig. 3. Fourier coefficients obtained by Fourier expansion of the measured line profiles.

tions in the microstructural features.

\subsection{Mechanical Properties}

The mechanical properties of the specimens were examined by tensile tests and hardness tests. The yield point,

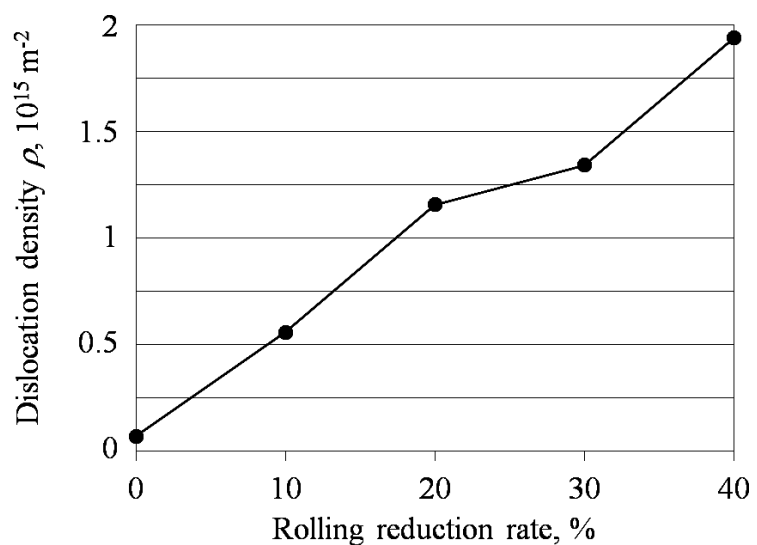

Fig. 4. Analyzed dislocation densities as a function of the rolling reduction rate.

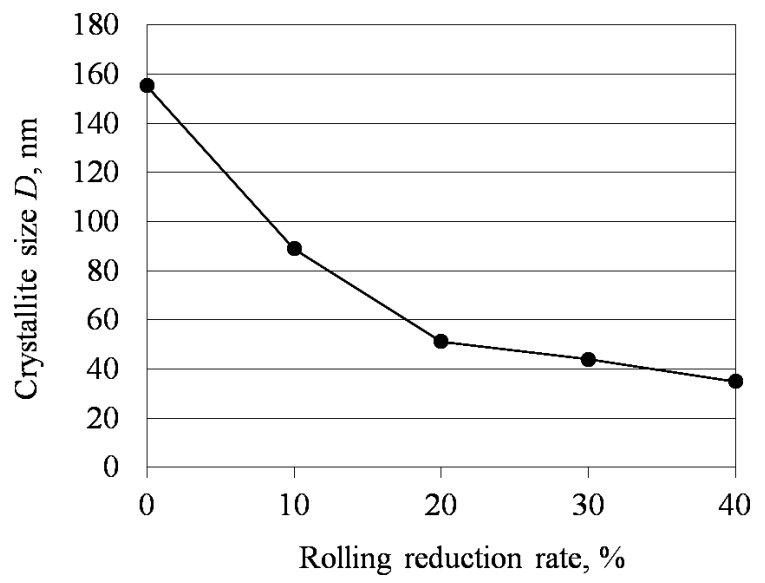

Fig. 5. Analyzed crystallite size as a function of the rolling reduction rate.

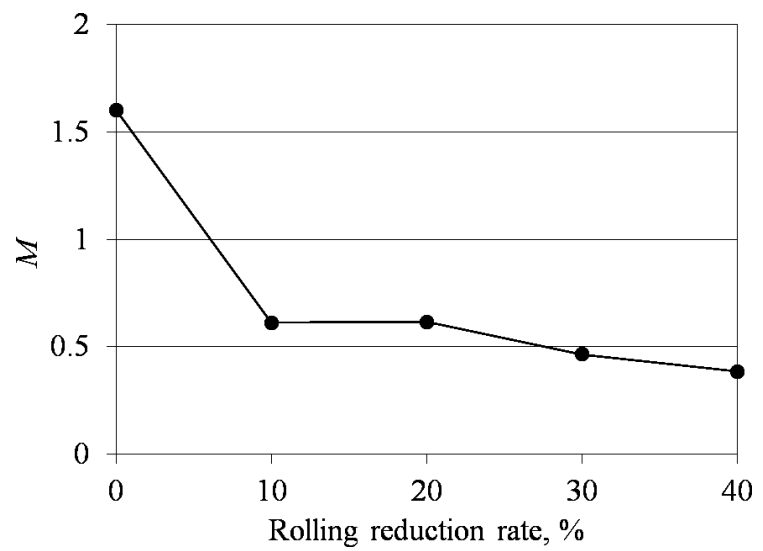

Fig. 6. Analyzed $M$ values as a function of the rolling reduction rate.

which indicates the starting point of plastic deformation, was not determinable for the cold-rolled specimens; the $0.2 \%$ proof stress was therefore used instead of the yield point. Figure 7 shows the obtained proof stress, tensile strength, and hardness for samples prepared at each rolling reduction rate: aforementioned values for the material in its initial state were 350,606 , and $178 \mathrm{MPa}$, respectively. After $40 \%$ cold-rolling, these values increased to 708,854 , and $258 \mathrm{MPa}$, respectively. All parameters increased with an increase in the rolling reduction rate. The rate of increase in 


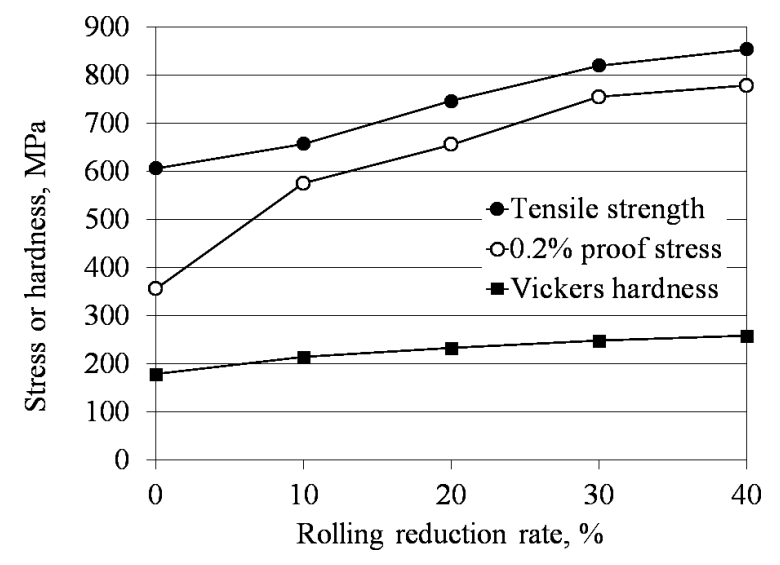

Fig. 7. Mechanical properties of cold-rolled specimens.

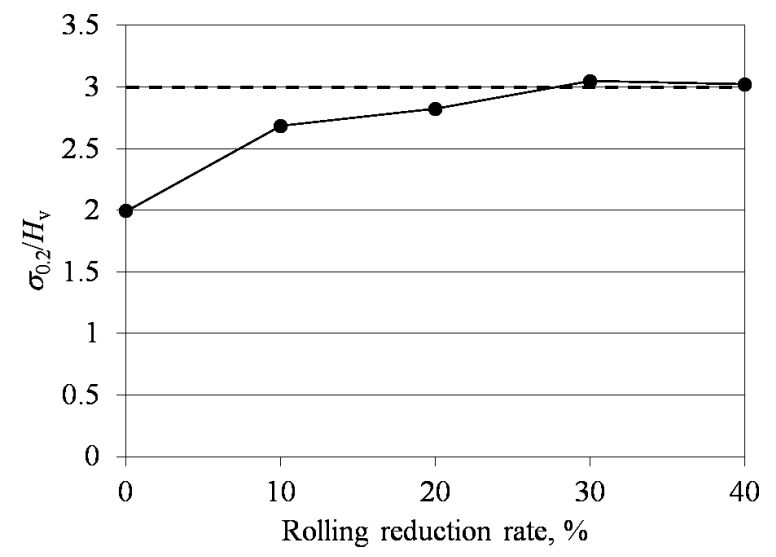

Fig. 8. Correlation between the proof stress and hardness of coldrolled specimens. A yield stress/hardness ratio of 3 indicates sufficient work-hardening.

the hardness, however, was lower than the rate of increase in the proof stress. Figure 8 shows the ratio of proof stress to hardness for samples prepared at each rolling reduction rate. The ratio was 2 in a sample that was not cold-rolled and increased to approximately 3 in a sample that was $40 \%$ coldrolled. This ratio is known, in general, to be approximately 3 . $^{14)}$ The results for the samples subjected to $30 \%$ and $40 \%$ rolling were also approximately 3 . In contrast, samples subjected to $0 \%$ to $20 \%$ rolling were less than 3 . This ratio, in general, approaches 3 when samples have been sufficiently work-hardened. ${ }^{15)}$ However, specimens subjected to reduction rates less than $30 \%$ have not been sufficiently workhardened and will therefore be further work-hardened under greater rolling reduction rates.

\subsection{Correlation between Microstructures and Mechani- cal Properties}

Here, we discuss correlations between the microstructural characters and mechanical properties. Flow stress and dislocation density are known to exhibit the following BaileyHirsch relationship: ${ }^{16)}$

$$
\tau=\tau_{0}+\alpha \mu b \sqrt{\rho}
$$

where, $\alpha$ and $\tau_{0}$ are constants, and $\mu$ is the shear modulus. Our results for flow shear stress are plotted in Fig. 9 as a function of the square root of the dislocation density, in accordance with Eq. (7), and the regression line was estimated by a least-squares method. When flow stresses were

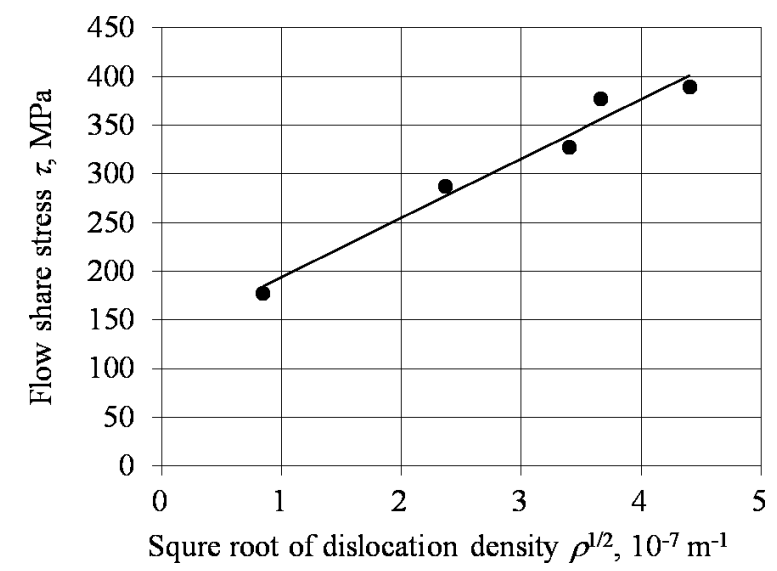

Fig. 9. Correlations between the square root of the dislocation density and the flow shear stress. The black circles denote the experimental results; the line denotes the linear regression result for the data.

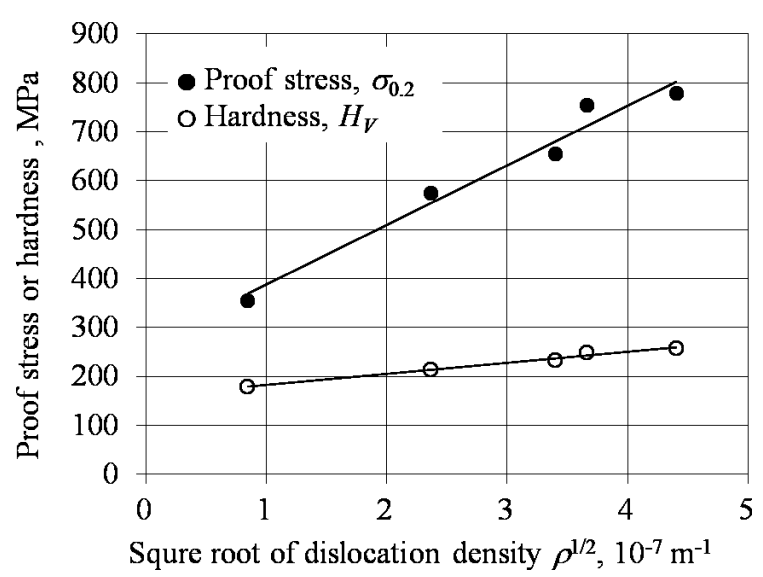

Fig. 10. Comparison of the correlations between the proof stress and hardness as functions of the square root of the dislocation density. The filled and open circles denote experimental results; the lines denote the results of linear regression of the data.

assumed to be half the proof stress values, $\alpha$ became 0.31 and $\tau_{0}$ became $133 \mathrm{MPa}$. This tendency agreed with the correlation shown in Eq. (7).

Figure 10 shows a plot of the proof stress and hardness as a function of the square root of the dislocation density. The correlation between the proof stress and the square root of the dislocation density was linear, as was the relationship between the flow shear stress and the square root of the dislocation density shown in Fig. 9. The correlation between the hardness and the square root of the dislocation density was also linear; however, the ratios between the proof stress and hardness $\left(\sigma_{0.2} / H_{\mathrm{v}}\right)$ were not constant, as shown in Fig. 8. On the other hand, if the dislocation density increases to infinity, the $\sigma_{0.2} / H_{\mathrm{v}}$ ratio increases to 5.4 due to the relationship shown in Fig. 10. The dislocation density, in reality, does not increase to infinity and saturates at $10^{16}-10^{17} \mathrm{~m}^{-2}$. In this study, both the proof stress and hardness almost reached saturation in the samples subjected to $40 \%$ rolling, even though the dislocation density did not saturate. These results indicate that work-hardening does not depend only on the total amount of dislocations; it also depends on other factors, such as the type and arrangement of dislocations. As shown in Figs. 5 and 6 , the crystallite sizes and $M$ values 
decreased with increases in the rolling reduction rate, particularly during the initial stage of rolling reduction. Consequently, these factors, which include variations in the crystallite size and the arrangement of dislocations, can affect the work-hardening.

\section{Conclusions}

Line profile analyses of cold-rolled carbon steels were conducted to evaluate microstructural features in nanoscale, such as dislocation density, crystallite size, and $M$ values. The dislocation density of the materials increased from $7 \times 10^{13}$ to $2 \times 10^{15} \mathrm{~m}^{-2}$ and the crystallite size decreased from 155 to $35 \mathrm{~nm}$ after they were subjected to $40 \%$ coldrolling. The component ratio of screw and edge dislocations was approximately $1: 1$ based on our evaluation of the $q$ value. The $M$ value that indicated interaction of dislocations value was substantially decreased during the initial stage of cold-rolling and subsequently slowly decreased as the rolling reduction rate was increased. The decrease of $M$ value means that interaction of dislocations becomes strong.

The proof stress, hardness, and tensile strength were increased by cold-rolling. The ratios between the proof stress and hardness were initially 2 and increased to approximately 3 after the cold-rolling process. The correlation between the microstructural characters and mechanical properties was demonstrated according to the Bailey-Hirsch relationship between flow shear stress and dislocation density. Variations in the proof stress and hardness as a function of the square root of the dislocation density suggest that not only the total amount of dislocations but also other factors, such as the crystallite size and the dislocation arrangement, affect the work-hardening process.

\section{Acknowledgement}

We appreciate the experimental efforts of two students of Tokyo City University, Shota Tsushima and Naoto Yamaguchi.

\section{REFERENCES}

1) B. E. Warren: X-ray diffraction, Dover Publications Inc, New York, (1990), 251.

2) G. K. Williamson and W. H. Hall: Acta Metall., 1 (1953), 22.

3) B. E. Warren and B. L. Averbach: J. Appl. Phys., 21 (1950), 595.

4) Y. Shibutani: Sosei no butsuri, Morikita Publishing, Tokyo, (2011), 68

5) T. Ungár and A. Borbély: Appl. Phys. Lett., 69 (1996), 3173.

6) T. Ungár, S. Ott, P. Sanders, A. Borbély and J. Weertman: Acta Mater., 46 (1998), 3693.

7) T. Ungár, L. Dragomir, Á. Révész and A. Borbély: J. Appl. Crystal$\log r ., 32$ (1999), 992.

8) I. Dragomir-Cernatescu, M. Gheorghe, N. Thadhani and R. L. Snyder: Powder Diffr., 48 (2005), 67.

9) M. Wilkens: Phys. Status Solid. A, 2 (1970), 359.

10) A. Borbély, J. D.-Cernatescu, C. Ribárik and T. Ungár: J. Appl. Crystallogr., 36 (2003), 160.

11) A. E. Lord, Jr. and D. N. Beshers: J. Appl. Phys., 36 (1965), 1620.

12) T. Ungár, J. Gubicza, G. Ribárik and A. Borbély: J. Appl. Crystal$\log r ., 34$ (2001), 298.

13) L. Balogh, D. W. Browna, P. Mosbrucker, F. Long and M. R. Daymond: Acta Mater., 60 (2012), 5567.

14) D. Tabor: Proc. R. Soc. A, 192 (1948), 247.

15) M. M. Chaudhri: Acta Mater., 46 (1998), 3047.

16) S. Kohda: Introduction to Metal Physics, Corona publishing, Tokyo, (1973), 343. 